ANNALES

POLONICI MATHEMATICI

$91.1(2007)$

\title{
Meromorphic functions sharing three values with finite weight
}

\author{
by AbHiJit BanerJee (Kalyani)
}

\begin{abstract}
Using the idea of weighted sharing we prove some theorems on uniqueness of meromorphic functions that share three values, which improve and supplement some results of Yi. Also we solve a problem recently raised by the present author [Austral. J. Math. Anal. Appl. 3 (2006)]. Examples are provided to show that some results are sharp.
\end{abstract}

1. Introduction and definitions. Let $f$ and $g$ be two nonconstant meromorphic functions defined in the open complex plane $\mathbb{C}$. If for some $a \in$ $\mathbb{C} \cup\{\infty\}, f$ and $g$ have the same set of $a$-points with the same multiplicities then we say that $f$ and $g$ share the value a $C M$ (counting multiplicities). If we do not take the multiplicities into account, then $f$ and $g$ are said to share the value a IM (ignoring multiplicities). The notation $T(r)$ denotes the maximum of $T(r, f)$ and $T(r, g)$.

We use $I$ to denote any set of $0<r<\infty$ of infinite linear measure, not necessarily the same at each occurrence. Though we do not explain the standard notation of value distribution theory because it is available in [3] we explain some notations which will be needed in the following.

Definition 1.1. Let $s$ be a positive integer. We denote by $\bar{N}(r, a ; f \mid=s)$ the reduced counting function of those $a$-points of $f$ whose multiplicity is exactly $s$, for $a \in \mathbb{C} \cup\{\infty\}$.

Definition $1.2([4,5])$. Let $s$ be a positive integer and $a \in \mathbb{C} \cup\{\infty\}$. We denote by $\bar{N}(r, a ; f \mid \geq s)$ the counting function of those $a$-points of $f$ whose multiplicities are greater than or equal to $s$, where each $a$-point is counted only once.

Ozawa [8] and Ueda [10] dealt with the problem of uniqueness of entire and meromorphic functions that share three distinct values. G. Brosch [2] improved the earlier results and proved the following theorem.

2000 Mathematics Subject Classification: Primary 30D35.

Key words and phrases: meromorphic functions, uniqueness, weighted sharing, shared set. 
Theorem A ([2]). Let $f$ and $g$ share $0,1, \infty$ CM. If

$$
\limsup _{\substack{r \rightarrow \infty \\ r \in I}} \frac{2 \bar{N}(r, 0 ; f)+2 \bar{N}(r, \infty ; f)-m(r, 1 ; g)}{T(r, f)}<1
$$

then either $f \equiv g$ or $f g \equiv 1$.

During the last few years a considerable amount of work has been devoted to the uniqueness problem for meromorphic functions sharing three values (cf. [7], [9], [11]-[13], [16]).

In 1998 H. X. Yi improved all the previous results and proved the following three theorems.

Theorem B ([14]). Let $f$ and $g$ share $1, \infty, 0$ CM. If

$$
\limsup _{\substack{r \rightarrow \infty \\ r \in I}} \frac{N(r, 0 ; f \mid=1)+N(r, \infty ; f \mid=1)-\frac{1}{2} m(r, 1 ; g)}{T(r, f)}<\frac{1}{2}
$$

then either $f \equiv g$ or $f g \equiv 1$.

Theorem $\mathrm{C}([14])$. Let $f$ and $g$ share $1, \infty C M$ and 0 IM. If

$$
\limsup _{\substack{r \rightarrow \infty \\ r \in I}} \frac{3 \bar{N}(r, 0 ; f)+2 \bar{N}(r, \infty ; f)-m(r, 1 ; g)}{T(r, f)}<1
$$

then either $f \equiv g$ or $f g \equiv 1$.

Theorem D ([14]). Let $f$ and $g$ share $0, \infty$ IM and 1 CM. If

$$
\limsup _{\substack{r \rightarrow \infty \\ r \in I}} \frac{3 \bar{N}(r, 0 ; f)+3 \bar{N}(r, \infty ; f)-m(r, 1 ; g)}{T(r, f)}<1
$$

then either $f \equiv g$ or $f g \equiv 1$.

To state the next results we have to introduce the notion of gradation of sharing known as weighted sharing.

Definition $1.3([4,5])$. Let $k$ be a nonnegative integer or infinity. For $a \in \mathbb{C} \cup\{\infty\}$ we denote by $E_{k}(a ; f)$ the set of all $a$-points of $f$ where an $a$-point of multiplicity $m$ is counted $m$ times if $m \leq k$ and $1+k$ times if $m>k$. If $E_{k}(a ; f)=E_{k}(a ; g)$, we say that $f, g$ share the value a with weight $k$.

The definition implies that if $f, g$ share a value $a$ with weight $k$ then $z_{0}$ is an $a$-point of $f$ with multiplicity $m(\leq k)$ if and only if it is an $a$-point of $g$ with multiplicity $m(\leq k)$, and $z_{0}$ is an $a$-point of $f$ with multiplicity $m(>k)$ if and only if it is an $a$-point of $g$ with multiplicity $n(>k)$, where $m$ is not necessarily equal to $n$.

We write $f, g$ share $(a, k)$ to mean that $f, g$ share the value $a$ with weight $k$. Clearly if $f, g$ share $(a, k)$ then $f, g$ share $(a, p)$ for all integers 
$p$ with $0 \leq p<k$. Also we note that $f, g$ share a value $a$ IM or CM if and only if $f, g$ share $(a, 0)$ or $(a, \infty)$ respectively.

In 2001 with the notion of weighted sharing of values the following two results were proved in [4].

THEOREM E ([4]). Let $f$ and $g$ be two nonconstant meromorphic functions sharing $(0,1),(\infty, 0)$ and $(1, \infty)$. If

$$
N(r, 0 ; f \mid=1)+4 \bar{N}(r, \infty ; f)<(\lambda+o(1)) T(r)
$$

for $r \in I$ and $0<\lambda<1 / 2$ then either $f \equiv g$ or $f g \equiv 1$.

Theorem $\mathrm{F}([4])$. Let $f$ and $g$ be two nonconstant meromorphic functions sharing $(0,1),(\infty, \infty)$ and $(1, \infty)$. If

$$
N(r, 0 ; f \mid=1)+N(r, \infty ; f \mid=1)<(\lambda+o(1)) T(r)
$$

for $r \in I$ and $0<\lambda<1 / 2$ then either $f \equiv g$ or $f g \equiv 1$.

Also in 2003 with the same notion of weighted sharing of values, improving Theorems $\mathrm{C}$ and $\mathrm{D}$, Lahiri proved the following two theorems.

Theorem $\mathrm{G}([6])$. Let $f$ and $g$ share $(0,0),(1,2),(\infty, \infty)$. If condition (1.1) holds then either $f \equiv g$ or $f g \equiv 1$.

Theorem $\mathrm{H}([6])$. Let $f$ and $g$ share $(0,0),(1,2),(\infty, 0))$. If condition (1.2) holds then either $f \equiv g$ or $f g \equiv 1$.

Recently in [1] the present author has improved Theorem H by relaxing the condition (1.2). In the same paper the author has also raised the problem of relaxing condition (1.1) in Theorem G. In this paper we will solve this last problem.

In $2003 \mathrm{Yi}$ [15] improved Theorems $\mathrm{E}$ and $\mathrm{F}$ by proving the following three theorems.

Theorem I ([15]). Let $f$ and $g$ share $(0,1),(\infty, 0),(1,5)$. If

$$
\limsup _{\substack{r \rightarrow \infty \\ r \in I}} \frac{N(r, 0 ; f \mid=1)+3 \bar{N}(r, \infty ; f)-\frac{1}{2} m(r, 1 ; g)}{T(r, f)}<\frac{1}{2}
$$

then either $f \equiv g$ or $f g \equiv 1$.

Theorem $\mathrm{J}([15])$. Let $f$ and $g$ share $(0,1),(\infty, 0),(1,3)$. If

$$
\limsup _{\substack{r \rightarrow \infty \\ r \in I}} \frac{N(r, 0 ; f \mid=1)+4 \bar{N}(r, \infty ; f)-\frac{1}{2} m(r, 1 ; g)}{T(r, f)}<\frac{1}{2}
$$

then either $f \equiv g$ or $f g \equiv 1$. 
Theorem K ([15]). Let $f$ and $g$ share $(0,1),(\infty, 2),(1,6)$. If

$$
\limsup _{\substack{r \rightarrow \infty \\ r \in I}} \frac{N(r, 0 ; f \mid=1)+N(r, \infty ; f \mid=1)-\frac{1}{2} m(r, 1 ; g)}{T(r, f)}<\frac{1}{2}
$$

then either $f \equiv g$ or $f g \equiv 1$.

In this paper we will improve Theorems I and $\mathrm{J}$ by weakening the conditions (1.3) and (1.4). We will also investigate the question of further reduction of the weight of the value 1 in Theorem $\mathrm{K}$.

We now state some more definitions.

DeFinition 1.4. Let $f$ and $g$ be two nonconstant meromorphic functions such that $f$ and $g$ share $(a, k)$ where $a \in \mathbb{C} \cup\{\infty\}$. Let $z_{0}$ be an $a$-point of $f$ with multiplicity $p$, and an $a$-point of $g$ with multiplicity $q$. We denote by $\bar{N}_{L}(r, a ; f)$ (resp. $\left.\bar{N}_{L}(r, a ; g)\right)$ the counting function of those $a$-points of $f$ and $g$ where $p>q$ (resp. $q>p)$, and by $\bar{N}_{E}^{(k+1}(r, a ; f)$ the counting function of those $a$-points of $f$ and $g$ where $p=q \geq k+1$. Each point in these counting functions is counted only once. In the same way we can define $\bar{N}_{E}^{(k+1}(r, a ; g)$. Clearly $\bar{N}_{E}^{(k+1}(r, a ; f) \equiv \bar{N}_{E}^{(k+1}(r, a ; g)$.

Definition $1.5([4,5])$. Let $f, g$ share a value $a$ IM. We denote by $\bar{N}_{*}(r, a ; f, g)$ the reduced counting function of those $a$-points of $f$ whose multiplicities differ from the multiplicities of the corresponding $a$-points of $g$. Clearly $\bar{N}_{*}(r, a ; f, g) \equiv \bar{N}_{*}(r, a ; g, f)$ and $\bar{N}_{*}(r, a ; f, g)=\bar{N}_{L}(r, a ; f)+$ $\bar{N}_{L}(r, a ; g)$.

2. Lemmas. In this section we present some lemmas which will be needed later on. Henceforth we shall denote by $H, \phi_{1}, \phi_{2}, \phi_{3}$ the following four functions:

$$
\begin{aligned}
& H=\frac{f^{\prime \prime}}{f^{\prime}}-\frac{2 f^{\prime}}{f-1}-\frac{g^{\prime \prime}}{g^{\prime}}+\frac{2 g^{\prime}}{g-1}, \\
& \phi_{1}=\frac{f^{\prime}}{f(f-1)}-\frac{g^{\prime}}{g(g-1)}=\left(\frac{f^{\prime}}{f-1}-\frac{g^{\prime}}{g-1}\right)-\left(\frac{f^{\prime}}{f}-\frac{g^{\prime}}{g}\right), \\
& \phi_{2}=\frac{f^{\prime}}{f-1}-\frac{g^{\prime}}{g-1}, \quad \phi_{3}=\frac{f^{\prime}}{f}-\frac{g^{\prime}}{g} .
\end{aligned}
$$

Lemma $2.1([4,6])$. If $f, g$ share $(0,0),(1,0),(\infty, 0)$ then

(i) $T(r, f) \leq 3 T(r, g)+S(r, f)$,

(ii) $T(r, g) \leq 3 T(r, f)+S(r, g)$.

Lemma 2.1 shows that $S(r, f)=S(r, g)$ and we denote their common value by $S(r)$. 
Lemma $2.2([14])$. Let $f, g$ share $(0,0),(1,0),(\infty, 0)$ and suppose $H \equiv 0$. Then $f, g$ share $(0, \infty),(1, \infty),(\infty, \infty)$.

Lemma $2.3([5])$. Let $f, g$ share $(1,1)$ and $H \not \equiv 0$. Then

$$
N(r, 1 ; f \mid=1)=N(r, 1 ; g \mid=1) \leq N(r, H)+S(r, f)+S(r, g) .
$$

Lemma $2.4([6])$. Let $f, g$ share $(0,0),(1,0),(\infty, 0)$ and $H \not \equiv 0$. Then

$$
\begin{aligned}
N(r, H) \leq & \bar{N}_{*}(r, 0 ; f, g)+\bar{N}_{*}(r, \infty ; f, g)+\bar{N}_{*}(r, 1 ; f, g) \\
& +\bar{N}_{0}\left(r, 0 ; f^{\prime}\right)+\bar{N}_{0}\left(r, 0 ; g^{\prime}\right),
\end{aligned}
$$

where $\bar{N}_{0}\left(r, 0 ; f^{\prime}\right)$ is the reduced counting function of those zeros of $f^{\prime}$ which are not the zeros of $f(f-1)$ and $\bar{N}_{0}\left(r, 0 ; g^{\prime}\right)$ is similarly defined.

LEMma 2.5. Let $f$ and $g$ be two nonconstant meromorphic functions sharing $(1, k)$, where $2 \leq k<\infty$. Then

$\bar{N}(r, 1 ; f \mid=2)+2 \bar{N}(r, 1 ; f \mid=3)+\cdots+(k-1) \bar{N}(r, 1 ; f \mid=k)$

$+k \bar{N}_{L}(r, 1 ; f)+(k+1) \bar{N}_{L}(r, 1 ; g)+k \bar{N}_{E}^{(k+1}(r, 1 ; f) \leq N(r, 1 ; g)-\bar{N}(r, 1 ; g)$.

Proof. Let $z_{0}$ be a 1-point of $f$ of multiplicity $p$, and a 1-point of $g$ of multiplicity $q$. We denote by $N_{1}(r), N_{2}(r)$ and $N_{3}(r)$ the counting functions of those 1-points of $f$ and $g$ when $k+1 \leq q<p, k+1 \leq q=p$ and $k+1 \leq p<q$ respectively. Each point in these counting functions is counted $q-k$ times.

Since $f, g$ share $(1, k)$, we have

$$
\begin{aligned}
N(r, 1 ; g)-\bar{N}(r, 1 ; g) & \\
= & \bar{N}(r, 1 ; f \mid=2)+2 \bar{N}(r, 1 ; f \mid=3)+\cdots \\
& +(k-1) \bar{N}(r, 1 ; f \mid=k)+(k-1) \bar{N}_{L}(r, 1 ; f) \\
& +(k-1) \bar{N}_{L}(r, 1 ; g)+(k-1) \bar{N}_{E}^{(k+1}(r, 1 ; f) \\
& +N_{1}(r)+N_{2}(r)+N_{3}(r) .
\end{aligned}
$$

Also we note that

$$
\begin{aligned}
& N_{1}(r) \geq \bar{N}_{L}(r, 1 ; f), \\
& N_{2}(r) \geq \bar{N}_{E}^{(k+1}(r, 1 ; f), \\
& N_{3}(r) \geq 2 \bar{N}_{L}(r, 1 ; g),
\end{aligned}
$$

Using (2.2)-(2.4) in (2.1) we deduce that

$$
\begin{aligned}
N(r, 1 ; g)-\bar{N}(r, 1 ; g) \geq & \bar{N}(r, 1 ; f \mid=2)+2 \bar{N}(r, 1 ; f \mid=3)+\cdots \\
& +(k-1) \bar{N}(r, 1 ; f \mid=k)+k \bar{N}_{L}(r, 1 ; f) \\
& +(k+1) \bar{N}_{L}(r, 1 ; g)+k \bar{N}_{E}^{(k+1}(r, 1 ; f) .
\end{aligned}
$$

This proves the lemma. 
Lemma 2.6. Let $f, g$ share $(0, l),(\infty, m),(1, k)$, where $2 \leq k<\infty$, and suppose $H \not \equiv 0$. Then

$$
\begin{aligned}
T(r, f) \leq & \bar{N}(r, 0 ; f)+\bar{N}(r, 0 ; g)+\bar{N}_{*}(r, 0 ; f, g)+\bar{N}(r, \infty ; f) \\
& +\bar{N}(r, \infty ; g)+\bar{N}_{*}(r, \infty ; f, g) \\
& -m(r, 1 ; g)-\bar{N}(r, 1 ; f \mid=3)-\cdots-(k-2) \bar{N}(r, 1 ; f \mid=k) \\
& -(k-2) \bar{N}_{L}(r, 1 ; f) \\
& -(k-1) \bar{N}_{L}(r, 1 ; g)-(k-1) \bar{N}_{E}^{(k+1}(r, 1 ; f)+S(r) .
\end{aligned}
$$

Proof. By the second fundamental theorem we get

$$
\begin{aligned}
T(r, f)+ & T(r, g) \\
\leq & \bar{N}(r, 0 ; f)+\bar{N}(r, \infty ; f)+\bar{N}(r, 0 ; g)+\bar{N}(r, \infty ; g) \\
& +\bar{N}(r, 1 ; f)+\bar{N}(r, 1 ; g)-N_{0}\left(r, 0 ; f^{\prime}\right)-N_{0}\left(r, 0 ; g^{\prime}\right) \\
& +S(r, f)+S(r, g) .
\end{aligned}
$$

Using Lemmas 2.3-2.5 we see that

$$
\begin{aligned}
& \bar{N}(r, 1 ; f)+\bar{N}(r, 1 ; g) \\
& =N(r, 1 ; f \mid=1)+\bar{N}(r, 1 ; f \mid=2)+\bar{N}(r, 1 ; f \mid=3) \\
& +\cdots+\bar{N}(r, 1 ; f \mid=k)+\bar{N}_{E}^{(k+1}(r, 1 ; f) \\
& +\bar{N}_{L}(r, 1 ; f)+\bar{N}_{L}(r, 1 ; g)+\bar{N}(r, 1 ; g) \\
& \leq \bar{N}_{*}(r, 0 ; f, g)+\bar{N}_{*}(r, \infty ; f, g)+\bar{N}_{L}(r, 1 ; f)+\bar{N}_{L}(r, 1 ; g) \\
& +\bar{N}(r, 1 ; f \mid=2)+\cdots+\bar{N}(r, 1 ; f \mid=k)+\bar{N}_{E}^{(k+1}(r, 1 ; f) \\
& +\bar{N}_{L}(r, 1 ; f)+\bar{N}_{L}(r, 1 ; g)+T(r, g)-m(r, 1 ; g) \\
& +O(1)-\bar{N}(r, 1 ; f \mid=2)-2 \bar{N}(r, 1 ; f \mid=3)-\cdots \\
& -(k-1) \bar{N}(r, 1 ; f \mid=k)-k \bar{N}_{E}^{(k+1}(r, 1 ; f)-k \bar{N}_{L}(r, 1 ; f) \\
& -(k+1) \bar{N}_{L}(r, 1 ; g)+\bar{N}_{0}\left(r, 0 ; f^{\prime}\right)+\bar{N}_{0}\left(r, 0 ; g^{\prime}\right)+S(r) \\
& \leq \bar{N}_{*}(r, 0 ; f, g)+\bar{N}_{*}(r, \infty ; f, g)+T(r, g)-m(r, 1 ; g) \\
& -\bar{N}(r, 1 ; f \mid=3)-2 \bar{N}(r, 1 ; f \mid=4)-\cdots \\
& -(k-2) \bar{N}(r, 1 ; f \mid=k)-(k-2) \bar{N}_{L}(r, 1 ; f) \\
& -(k-1) \bar{N}_{L}(r, 1 ; g)-(k-1) \bar{N}_{E}^{(k+1}(r, 1 ; f) \\
& +\bar{N}_{0}\left(r, 0 ; f^{\prime}\right)+\bar{N}_{0}\left(r, 0 ; g^{\prime}\right)+S(r)
\end{aligned}
$$

From (2.5) and (2.6) the lemma follows. 
Lemma 2.7. Let $f, g$ share $(0,1),(1,2),(\infty, 1)$. If $f \not \equiv g$ and $\bar{N}(r, \infty$; $f \mid \geq 2)=\bar{N}(r, \infty ; g \mid \geq 2)=S(r)$ then $\bar{N}(r, a ; f \mid \geq 2)=\bar{N}(r, a ; g \mid \geq 2)=S(r)$ for $a=0,1$.

Proof. We prove $\bar{N}(r, a ; f \mid \geq 2)=S(r)$ for $a=0,1$ because the case of $g$ is similar.

We suppose that $\bar{N}(r, a ; f) \neq S(r)$ for $a=0,1$ because otherwise the assertion is trivial. Since $f \not \equiv g$, it follows that $\phi_{i} \not \equiv 0$ for $i=2,3$. Now

$$
\begin{aligned}
\bar{N}(r, 0 ; f \mid \geq 2) & \leq N\left(r, 0 ; \phi_{2}\right) \\
& \leq T\left(r, \phi_{2}\right)+O(1)=N\left(r, \infty ; \phi_{2}\right)+S(r) \\
& \leq \bar{N}(r, 1 ; f \mid \geq 3)+\bar{N}(r, \infty ; f \mid \geq 2)+S(r) \\
& =\bar{N}(r, 1 ; f \mid \geq 3)+S(r) .
\end{aligned}
$$

Again

$$
\begin{aligned}
2 \bar{N}(r, 1 ; f \mid \geq 3) & \leq \bar{N}(r, 1 ; f \mid \geq 3)+\bar{N}(r, 1 ; f \mid \geq 2) \\
& \leq N\left(r, 0 ; \phi_{3}\right) \leq N\left(r, \infty ; \phi_{3}\right)+S(r) \\
& \leq \bar{N}(r, 0 ; f \mid \geq 2)+\bar{N}(r, \infty ; f \mid \geq 2)+S(r) \\
& =\bar{N}(r, 0 ; f \mid \geq 2)+S(r) .
\end{aligned}
$$

From (2.7) and (2.8) we get $\bar{N}(r, 0 ; f \mid \geq 2)=S(r)$ and hence from (2.8) we get $\bar{N}(r, 1 ; f \mid \geq 2)=S(r)$. This proves the lemma.

Lemma 2.8. Let $f, g$ share $(0,1),(1,1),(\infty, 2)$. If $f \not \equiv g$ and $\bar{N}(r, 1$; $f \mid \geq 2)=\bar{N}(r, 1 ; g \mid \geq 2)=S(r)$ then $\bar{N}(r, a ; f \mid \geq 2)=\bar{N}(r, a ; g \mid \geq 2)=S(r)$ for $a=0, \infty$.

Proof. We prove $\bar{N}(r, a ; f \mid \geq 2)=S(r)$ for $a=0, \infty$ because the case of $g$ is similar.

We suppose that $\bar{N}(r, a ; f) \neq S(r)$ for $a=0, \infty$ because otherwise the assertion is trivial. Since $f \not \equiv g$, it follows that $\phi_{i} \not \equiv 0$ for $i=1,2$. Now

$$
\begin{aligned}
2 \bar{N}(r, \infty ; f \mid \geq 3) & \leq \bar{N}(r, \infty ; f \mid \geq 3)+\bar{N}(r, \infty ; f \mid \geq 2) \\
& \leq N\left(r, 0 ; \phi_{1}\right) \leq N\left(r, \infty ; \phi_{1}\right)+S(r) \\
& \leq \bar{N}(r, 0 ; f \mid \geq 2)+\bar{N}(r, 1 ; f \mid \geq 2)+S(r) \\
& =\bar{N}(r, 0 ; f \mid \geq 2)+S(r) .
\end{aligned}
$$

Again

$$
\begin{aligned}
\bar{N}(r, 0 ; f \mid \geq 2) & \leq N\left(r, 0 ; \phi_{2}\right)=N\left(r, \infty ; \phi_{2}\right)+S(r) \\
& \leq \bar{N}(r, \infty ; f \mid \geq 3)+\bar{N}(r, 1 ; f \mid \geq 2)+S(r) \\
& =\bar{N}(r, \infty ; f \mid \geq 3)+S(r) .
\end{aligned}
$$

From (2.9) and (2.10) we get $\bar{N}(r, \infty ; f \mid \geq 3)=S(r)$. Then from (2.10) we get 
$\bar{N}(r, 0 ; f \mid \geq 2)=S(r)$ and hence using (2.9) we have $\bar{N}(r, \infty ; f \mid \geq 2)=S(r)$. This proves the lemma.

Lemma 2.9. Let $f, g$ share $(0,1),(1, m),(\infty, k)$ and $\bar{N}(r, 0 ; f \mid \geq 2)=$ $\bar{N}(r, 0 ; g \mid \geq 2)=S(r)$. If $f \not \equiv g$ and $m k-1>0$ then $\bar{N}(r, a ; f \mid \geq 2)=$ $\bar{N}(r, a ; g \mid \geq 2)=S(r)$ for $a=1, \infty$.

Proof. We prove $\bar{N}(r, a ; f \mid \geq 2)=S(r)$ for $a=1, \infty$ because the case of $g$ is similar.

We suppose that $\bar{N}(r, a ; f) \neq S(r)$ for $a=1, \infty$ because otherwise the assertion is trivial. Since $f \not \equiv g$, it follows that $\phi_{i} \not \equiv 0$ for $i=1,3$. Now

$$
\begin{aligned}
m \bar{N}(r, 1 ; f \mid \geq m & +1) \\
& \leq(m-1) \bar{N}(r, 1 ; f \mid \geq 1+m)+\bar{N}(r, 1 ; f \mid \geq 2) \\
& \leq N\left(r, 0 ; \phi_{3}\right) \leq N\left(r, \infty ; \phi_{3}\right)+S(r) \\
& \leq \bar{N}(r, \infty ; f \mid \geq k+1)+\bar{N}(r, 0 ; f \mid \geq 2)+S(r) \\
& =\bar{N}(r, \infty ; f \mid \geq k+1)+S(r) .
\end{aligned}
$$

Also

$$
\begin{aligned}
k \bar{N}(r, \infty ; f \mid \geq & k+1) \\
& \leq(k-1) \bar{N}(r, \infty ; f \mid \geq k+1)+\bar{N}(r, \infty ; f \mid \geq 2) \\
& \leq N\left(r, 0 ; \phi_{1}\right) \leq N\left(r, \infty ; \phi_{1}\right)+S(r) \\
& \leq \bar{N}(r, 1 ; f \mid \geq m+1)+\bar{N}(r, 0 ; f \mid \geq 2)+S(r) \\
& =\bar{N}(r, 1 ; f \mid \geq m+1)+S(r) .
\end{aligned}
$$

From (2.11) and (2.12) we get $(m-1 / k) \bar{N}(r, 1 ; f \mid \geq m+1) \leq S(r)$, i.e. $\bar{N}(r, 1 ; f \mid \geq m+1)=S(r)$. Then from $(2.12)$ we obtain $\bar{N}(r, \infty ; f \mid \geq 2)=$ $S(r)$. Again from $(2.11)$ we get $\bar{N}(r, 1 ; f \mid \geq 2)=S(r)$. This completes the proof of the lemma.

Lemma 2.10 ([15]). Let $f, g$ share $(0,1),(\infty, 0),(1, k)$ where $k \geq 2$ is a positive integer or infinity. Then

$$
\begin{aligned}
\bar{N}(r, 0 ; f \mid \geq 2) & \leq \bar{N}(r, \infty ; f)+\bar{N}(r, 1 ; f \mid \geq k+1)+S(r), \\
\bar{N}(r, 1 ; f \mid \geq k+1) & \leq \frac{2}{k-1} \bar{N}(r, \infty ; f)+S(r) .
\end{aligned}
$$

Lemma 2.11. Let $f, g$ share $(0,1),(1,1),(\infty, 1)$ and $\bar{N}(r, a ; f \mid \geq 2)=$ $S(r)$, where $a=0,1, \infty$ and $H \not \equiv 0$. Then

$$
T(r, f) \leq 2 N(r, 0 ; f \mid=1)+2 N(r, \infty ; f \mid=1)-m(r, 1 ; g)+S(r) .
$$

Proof. By the second fundamental theorem and Lemmas 2.3 and 2.4 we see that 


$$
\begin{aligned}
T(r, f)+T(r, g) \leq & 2 N(r, 0 ; f \mid=1)+2 \bar{N}(r, 0 ; f \mid \geq 2)+2 N(r, \infty ; f \mid=1) \\
& +2 \bar{N}(r, \infty ; f \mid \geq 2)+N(r, 1 ; f \mid=1)+N(r, 1 ; g) \\
& -N_{0}\left(r, 0 ; f^{\prime}\right)-N_{0}\left(r, 0 ; g^{\prime}\right)+S(r) \\
\leq & 2 N(r, 0 ; f \mid=1)+2 N(r, \infty ; f \mid=1)+\bar{N}(r, 0 ; f \mid \geq 2) \\
& +\bar{N}(r, \infty ; f \mid \geq 2)+\bar{N}(r, 1 ; f \mid \geq 2)+T(r, g) \\
& -m(r, 1 ; g)+O(1)+S(r)
\end{aligned}
$$

i.e.

$$
T(r, f) \leq 2 N(r, 0 ; f \mid=1)+2 N(r, \infty ; f \mid=1)-m(r, 1 ; g)+S(r)
$$

3. Theorems. In this section we state the main theorems of the paper.

THEOREM 3.1. Let $f$ and $g$ be two nonconstant meromorphic functions sharing $(0,0),(\infty, \infty),(1,2)$. If

$$
\limsup _{\substack{r \rightarrow \infty \\ r \in I}} \frac{3 \bar{N}(r, 0 ; f)+2 \bar{N}(r, \infty ; f)-m(r, 1 ; g)-\bar{N}_{L}(r, 1 ; g)}{T(r, f)}<1
$$

then either $f \equiv g$ or $f g \equiv 1$.

THEOREM 3.2. Let $f$ and $g$ be two nonconstant meromorphic functions sharing $(0,1),(\infty, 0),(1,5)$. If

$$
\limsup _{\substack{r \rightarrow \infty \\ r \in I}} \frac{N(r, 0 ; f \mid=1)+3 \bar{N}(r, \infty ; f)-\frac{1}{2} m(r, 1 ; g)-\frac{1}{2} \bar{N}_{L}(r, 1 ; g)}{T(r, f)}<\frac{1}{2}
$$

then either $f \equiv g$ or $f g \equiv 1$.

THEOREM 3.3. Let $f$ and $g$ be two nonconstant meromorphic functions sharing $(0,1),(\infty, 0),(1,3)$. If

$$
\limsup _{\substack{r \rightarrow \infty \\ r \in I}} \frac{N(r, 0 ; f \mid=1)+4 \bar{N}(r, \infty ; f)-\frac{1}{2} m(r, 1 ; g)-\frac{1}{2} \bar{N}_{L}(r, 1 ; g)}{T(r, f)}<\frac{1}{2}
$$

then either $f \equiv g$ or $f g \equiv 1$.

THEOREM 3.4. Let $f$ and $g$ be two nonconstant meromorphic functions sharing $(0,1),(\infty, 1),(1,2)$. If $\bar{N}(r, \infty ; f \mid \geq 2)=\bar{N}(r, \infty ; g \mid \geq 2)=S(r)$ and

$$
\limsup _{\substack{r \rightarrow \infty \\ r \in I}} \frac{N(r, 0 ; f \mid=1)+N(r, \infty ; f \mid=1)-\frac{1}{2} m(r, 1 ; g)}{T(r, f)}<\frac{1}{2}
$$

then either $f \equiv g$ or $f g \equiv 1$. 
THEOREM 3.5. Let $f$ and $g$ be two nonconstant meromorphic functions sharing $(0,1),(\infty, 2),(1,1)$. If $\bar{N}(r, 1 ; f \mid \geq 2)=\bar{N}(r, 1 ; g \mid \geq 2)=S(r)$ and

$$
\limsup _{\substack{r \rightarrow \infty \\ r \in I}} \frac{N(r, 0 ; f \mid=1)+N(r, \infty ; f \mid=1)-\frac{1}{2} m(r, 1 ; g)}{T(r, f)}<\frac{1}{2}
$$

then either $f \equiv g$ or $f g \equiv 1$.

THEOREM 3.6. Let $f$ and $g$ be two nonconstant meromorphic functions sharing $(0,1),(\infty, k),(1, m)$, where $m k-1>0$. If $\bar{N}(r, 0 ; f \mid \geq 2)=\bar{N}(r, 0$; $g \mid \geq 2)=S(r)$ and

$$
\limsup _{\substack{r \rightarrow \infty \\ r \in I}} \frac{N(r, 0 ; f \mid=1)+N(r, \infty ; f \mid=1)-\frac{1}{2} m(r, 1 ; g)}{T(r, f)}<\frac{1}{2}
$$

then either $f \equiv g$ or $f g \equiv 1$.

ExAmple 1. Let $f=\left(1-e^{z}\right)^{3}, g=3\left(e^{z}-1\right) / e^{2 z}$. Clearly $f, g$ share $(0,0),(\infty, \infty)$ and $(1, \infty)$. Here $\bar{N}_{L}(r, 1 ; g)=0$. Also $T(r, f)=3 T\left(r, e^{z}\right)+$ $O(1), T(r, g)=2 T\left(r, e^{z}\right)+O(1)$ and $\bar{N}(r, 0 ; f) \sim T\left(r, e^{z}\right), \bar{N}(r, \infty ; f)=0$, $N(r, 1 ; g) \sim 2 T\left(r, e^{z}\right)$ but neither $f \equiv g$ nor $f g \equiv 1$. So the condition (3.1) in Theorem 3.1 is sharp.

ExAmple 2. Let $f=e^{z}-e^{2 z}, g=e^{-z}-e^{-2 z}$. Clearly $f, g$ share $(0, \infty),(\infty, \infty)$ and $(1, \infty)$. Here $N(r, 0 ; f \mid=1) \sim T\left(r, e^{z}\right), \bar{N}(r, \infty ; f)=0$, $\bar{N}_{L}(r, 1 ; g)=0$. Again $T(r, f)=2 T\left(r, e^{z}\right)+O(1), T(r, g)=2 T\left(r, e^{z}\right)+O(1)$, $N(r, 1 ; g) \sim 2 T\left(r, e^{z}\right)$ but neither $f \equiv g$ nor $f g \equiv 1$. So the conditions $(3.2)$ and (3.3) in Theorems 3.2 and 3.3 respectively are sharp.

ExAmPle 3. Let $f=e^{2 z} /\left(e^{z}-1\right), g=1 / e^{z}\left(1-e^{z}\right)$. It is easy to see that $f, g$ share $(0, \infty),(\infty, \infty)$ and $(1, \infty)$. Also here $\bar{N}(r, 0 ; f \mid \geq 2)=$ $\bar{N}(r, 0 ; g \mid \geq 2)=\bar{N}(r, 1 ; f \mid \geq 2)=\bar{N}(r, 1 ; g \mid \geq 2)=\bar{N}(r, \infty ; f \mid \geq 2)=\bar{N}(r, \infty ;$ $g \mid \geq 2)=0$. Also $T(r, f)=2 T\left(r, e^{z}\right)+O(1), T(r, g)=2 T\left(r, e^{z}\right)+O(1)$, $N(r, \infty ; f \mid=1) \sim T\left(r, e^{z}\right), N(r, 0 ; f \mid=1)=0, N(r, 1 ; g) \sim 2 T\left(r, e^{z}\right)$ but neither $f \equiv g$ nor $f g \equiv 1$. So the condition (3.4) in Theorems $3.4-3.6$ is the best possible.

EXAMPLE 4. Let $f=e^{z}+1, g=e^{-z}+1$.

ExAmple 5. Let $f=1 /\left(1-e^{z}\right), g=e^{z} /\left(1-e^{z}\right)$.

From Examples $4-5$ it is also easy to see that condition (3.4) in Theorems $3.4-3.6$ is the best possible.

\section{Proofs of the theorems}

Proof of Theorem 3.1. Since $f, g$ share $(0,0),(\infty, \infty)$, it follows that $\bar{N}_{*}(r, \infty ; f, g) \equiv 0$ and $\bar{N}_{*}(r, 0 ; f, g) \leq \bar{N}(r, 0 ; f)$. Suppose $H \not \equiv 0$. Then from 
Lemma 2.6, for $k=2$ we get

$$
T(r, f) \leq 3 \bar{N}(r, 0 ; f)+2 \bar{N}(r, \infty ; f)-\bar{N}_{L}(r, 1 ; g)-m(r, 1 ; g)+S(r),
$$

which contradicts (3.1). So $H \equiv 0$. Hence by Lemma 2.2, $f$ and $g$ share $(0, \infty),(1, \infty),(\infty, \infty)$. So $\bar{N}_{L}(r, 1 ; g) \equiv 0$. Now by Theorem A the theorem follows.

Proof of Theorem 3.2. Since $f, g$ share $(0,1),(\infty ; 0)$, we have $N_{*}(r, 0$; $f, g) \leq \bar{N}(r, 0 ; f \mid \geq 2)$ and $\bar{N}_{*}(r, \infty ; f, g) \leq \bar{N}(r, \infty ; f)$. Suppose $H \not \equiv 0$. Then from Lemmas 2.6 and 2.10 we get, for $k=5$,

$$
\begin{aligned}
T(r, f) \leq & 2 \bar{N}(r, 0 ; f)+\bar{N}(r, 0 ; f \mid \geq 2)+3 \bar{N}(r, \infty ; f)-3 \bar{N}(r, 1 ; f \mid \geq 6) \\
& \quad-\bar{N}_{L}(r, 1 ; g)-m(r, 1 ; g)+S(r) \\
= & 2 N(r, 0 ; f \mid=1)+3 \bar{N}(r, 0 ; f \mid \geq 2)+3 \bar{N}(r, \infty ; f) \\
& \quad-3 \bar{N}(r, 1 ; f \mid \geq 6)-\bar{N}_{L}(r, 1 ; g)-m(r, 1 ; g)+S(r) \\
\leq & 2 N(r, 0 ; f \mid=1)+6 \bar{N}(r, \infty ; f)-\bar{N}_{L}(r, 1 ; g)-m(r, 1 ; g)+S(r),
\end{aligned}
$$

which contradicts (3.2). So $H \equiv 0$. Hence by Lemma $2.2, f$ and $g$ share $(0, \infty),(1, \infty),(\infty, \infty)$. So $\bar{N}_{L}(r, 1 ; g) \equiv 0$. Now by Theorem B the theorem follows.

Proof of Theorem 3.3. We omit the proof since it can be carried out along the lines of the proof of Theorem 3.2.

Proof of Theorem 3.4. Suppose $H \not \equiv 0$. Then $f \not \equiv g$. So from Lemmas 2.7 and 2.11 and the assumption of the theorem we derive a contradiction. So $H \equiv 0$. Hence the theorem follows from Lemma 2.2 and Theorem B.

Proof of Theorem 3.5. We omit the proof since using Lemmas 2.8 and 2.11 the proof can be carried out along the lines of the proof of Theorem 3.4 .

Proof of Theorem 3.6. Use Lemmas 2.9 and 2.11 and proceed in the same manner as in the proof of Theorem 3.4.

5. Some remarks. In $2003 \mathrm{Yi}$ proved the following theorems.

THEOREM L ([15]). Let $f$ and $g$ be two nonconstant meromorphic functions sharing $(0,0),(\infty, 1),(1,5)$. If

$$
\limsup _{\substack{r \rightarrow \infty \\ r \in I}} \frac{3 \bar{N}(r, 0 ; f)+N(r, \infty ; f \mid=1)-\frac{1}{2} m(r, 1 ; g)}{T(r, f)}<\frac{1}{2}
$$

then either $f \equiv g$ or $f g \equiv 1$. 
TheOrem M ([15]). Let $f$ and $g$ be two nonconstant meromorphic functions sharing $(0,0),(\infty, 1),(1,3)$. If

$$
\limsup _{\substack{r \rightarrow \infty \\ r \in I}} \frac{4 \bar{N}(r, 0 ; f)+N(r, \infty ; f \mid=1)-\frac{1}{2} m(r, 1 ; g)}{T(r, f)}<\frac{1}{2}
$$

then either $f \equiv g$ or $f g \equiv 1$.

As a consequence of Theorems 3.2 and 3.3 we improve Theorems L and $\mathrm{M}$.

TheOREM 5.1. Let $f$ and $g$ be two nonconstant meromorphic functions sharing $(0,0),(\infty, 1),(1,5)$. If

$$
\limsup _{\substack{r \rightarrow \infty \\ r \in I}} \frac{3 \bar{N}(r, 0 ; f)+N(r, \infty ; f \mid=1)-\frac{1}{2} m(r, 1 ; g)-\frac{1}{2} \bar{N}_{L}(r, 1 ; g)}{T(r, f)}<\frac{1}{2}
$$

then either $f \equiv g$ or $f g \equiv 1$.

Proof. Let

$$
F=1 / f, \quad G=1 / g
$$

It is clear that

$$
T(r, f)=T(r, F)+O(1)
$$

and

$$
m(r, 1 ; g)=m(r, 1 ; G)+O(1)
$$

From the given condition and by $(5.1)-(5.3)$ we get

$$
\limsup _{\substack{r \rightarrow \infty \\ r \in I}} \frac{N(r, 0 ; F \mid=1)+3 \bar{N}(r, \infty ; F)-\frac{1}{2} m(r, 1 ; G)-\frac{1}{2} \bar{N}_{L}(r, 1 ; G)}{T(r, F)}<\frac{1}{2}
$$

Since $f, g$ share $(0,0),(\infty, 1)$ and $(1,5)$, from $(5.1)$ it follows that $F, G$ share $(0,1),(\infty, 0)$ and $(1,5)$. So by Theorem 3.2 we get $F \equiv G$ or $F G \equiv 1$, from which the theorem follows.

THEOREM 5.2. Let $f$ and $g$ be two nonconstant meromorphic functions sharing $(0,0),(\infty, 1),(1,3)$. If

$$
\limsup _{\substack{r \rightarrow \infty \\ r \in I}} \frac{4 \bar{N}(r, 0 ; f)+N(r, \infty ; f \mid=1)-\frac{1}{2} m(r, 1 ; g)-\frac{1}{2} \bar{N}_{L}(r, 1 ; g)}{T(r, f)}<\frac{1}{2}
$$

then either $f \equiv g$ or $f g \equiv 1$.

Proof. Use Theorem 3.3 and proceed in the same manner as in the proof of Theorem 5.1.

Acknowledgements. The author is grateful to Prof. H. X. Yi for supplying him the electronic file of the paper [15]. 


\section{References}

[1] A. Banerjee, Uniqueness of meromorphic functions that share three values, Austral. J. Math. Anal. Appl. 3 (2006), Art. 1, 10 pp.

[2] G. Brosch, Eindeutigkeitssätze für meromorphe Funktionen, Thesis, Technical Univ. of Aachen, 1989.

[3] W. K. Hayman, Meromorphic Functions, Clarendon Press, Oxford, 1964.

[4] I. Lahiri, Weighted sharing and uniqueness of meromorphic functions, Nagoya Math. J. 161 (2001), 193-206.

[5] -, Weighted value sharing and uniqueness of meromorphic functions, Complex Variables 46 (2001), 241-253.

[6] -, Meromorphic functions sharing three values, Southeast Asian Bull. Math. 26 (2003), 961-966.

[7] E. Mues and M. Reinders, Meromorphic functions sharing one value and unique range sets, Kodai Math. J. 18 (1995), 515-522.

[8] M. Ozawa, Unicity theorems for entire functions, J. Anal. Math. 30 (1976), 411-420.

[9] N. Terglane, Identitätssätze in $\mathbb{C}$ meromorpher Funktionen als Ergebnis von Werteteilung, Diplomarbeit, RWTH Aachen, 1989.

[10] H. Ueda, Unicity theorems for meromorphic or entire functions II, Kodai Math. J. 6 (1983), 26-36.

[11] - On the zero-one-pole set of a meromorphic function II, ibid. 13 (1990), 134-142.

[12] H. X. Yi, Meromorphic functions sharing three values, Chinese Ann. Math. Ser. A 9 (1988), 433-440.

[13] —, Unicity theorems for meromorphic functions that share three values, Kodai Math. J. 18 (1995), 300-314.

[14] -, Meromorphic functions that share three values, Bull. Hong Kong Math. Soc. 2 (1998), 55-64.

[15] -, On some results of Lahiri, J. Math. Anal. Appl. 284 (2003), 481-495.

[16] Q. C. Zhang, Meromorphic functions sharing three values, Indian J. Pure Appl. Math. 30 (1999), 667-682.

Department of Mathematics

Kalyani Government Engineering College

West Bengal 741235, India

E-mail: abanerjee_kal@yahoo.co.in, abanerjee@mail15.com, abanerjee_kal@rediffmail.com

Received 11.8.2006

and in final form 13.12.2006 